\title{
Clinical Signs and Symptoms of a Patient with Abdominal Pain: Case Report of a Rare Ileus Presentation
}

\author{
Erkan Güvenç ${ }^{1}$, Ömer Engin², Mustafa Küçük ${ }^{1}$, Burcu Tanay Demirdöven ${ }^{1}$, \\ Hilmi Güngör ${ }^{2}$
}

${ }^{1}$ Buca Seyfi Demirsoy State Hospital, Department of Emergency Medicine, İzmir, Turkey

${ }^{2}$ Buca Seyfi Demirsoy State Hospital, Department of Surgery, İzmir, Turkey

\begin{abstract}
In this report, we tried to discuss the pathologic mechanisms of abdominal pain and describe correct modalities of diagnosis by defining clinical findings and symptoms of patients admitted with abdominal pain.

Evaluating abdominal pain requires an approach that relies on the probability of disease, patient history, physical examination, laboratory tests, and imaging studies. The location of pain is a useful starting point and will guide further evaluation.
\end{abstract}

Keywords: Abdominal pain, ileus, inguinal hernia, Orchiectomy

\section{INTRODUCTION}

In 2004, 350,432 peptic ulcers, gastritis, duodenitis, appendicitis, intestinal obstruction, and infertile patients, all of whom were abdominal pain, were hospitalized according to the Turkish Statistical Institute (TSI). Among all the hospitalized patients, the rate of hospitalization due to these diagnoses was $5,73 \%$ in $2004.30 \%$ of the patients with abdominal pain still has not been diagnosed properly [1].

Abdominal hernias remain a common and annoying problem for both clinicians and radiologists. Unlike inguinal hernias that are clinically diagnosed and require minor surgical intervention, the diagnosis of internal herniation can be difficult and may require more extensive surgical intervention. Inguinal hernias carry the risk of serious complications. Additionally, it is important to define structures within a hernia sac for performing a proper surgery.

Therefore, accurate preoperative diagnosis can lead to more appropriate surgical management. Abdominal imaging is often the first clue for correct diagnosis. In the past, the diagnosis of a hernia was either made clinically, with plain radiographs, or with barium studies [2]. Currently, specific diagnoses are made more frequently by $\mathrm{CT}$ in patients with nonspecific abdominal complaints [3]. CT findings may be subtle or confusing; therefore, familiarity with a broad range of imaging results can help the radiologist make an early and specific diagnosis of abdominal hernia.

\section{CASE}

35-year-old man presented to our emergency department with abdominal pain for a 2 days. The patient's vital signs improved BP: 120/60 mm Hg, HR: 86 beats / min, RR: 18 / min, SpO2: 98\%, fever: it was $36.6^{\circ} \mathrm{C}$. The abdominal physical examination findings are consistent with distended abdominal and tenderness. Right strangulated hernia was found. Right testis was not in the scrotum. Right testis was in the inguinal canal congenitally.

In the laboratory tests of the patient is; WBC: 18.200, hemoglobin 14.9, hematocrit: 44.9, MCV: 73.7. Glucose: 121, Urea 33.2, Creat: 0.94, AST: 13.7, ALT: 23.6, Na: 139 K: 4.44, CI: 98, metalic images, minimal intra-abdominal fluid and intestinal obstruction are observed at abdominal CT [Figure 1,2]. 


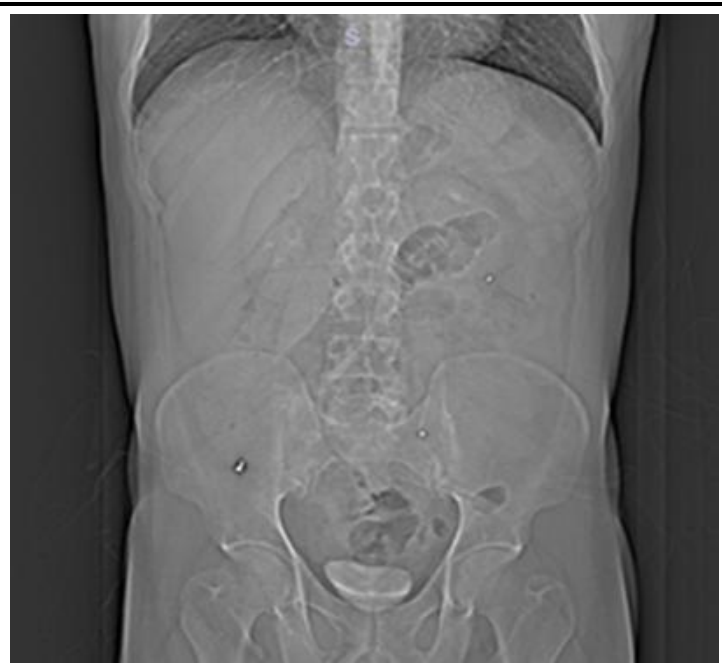

Figure 1. Metalic images (Coronal Plane)

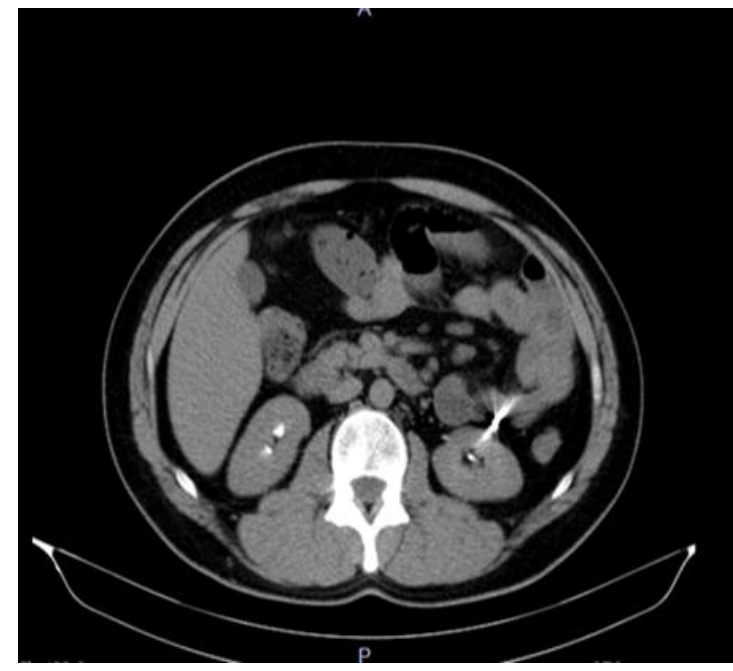

Figure 2. Metalic images and minimal intra-abdominal fluid (Transverse Plane)

The patient was asked what the metalic images could be. It was understood that his tooth filling droped and couldn't find it, so he may have swallowed it. Also he said that a few days ago he hunted rabbits with small shots and ate them.

The cause of the intestinal obstruction was inguinal herniation [Figure 3]. After the sedation the herniation was reduced and the symptoms reduced. He was operated electively by the general surgeon and urologist for the right testicle which was in the inguinal canal. The testical was nonfunctional so orchiectomy was performed.

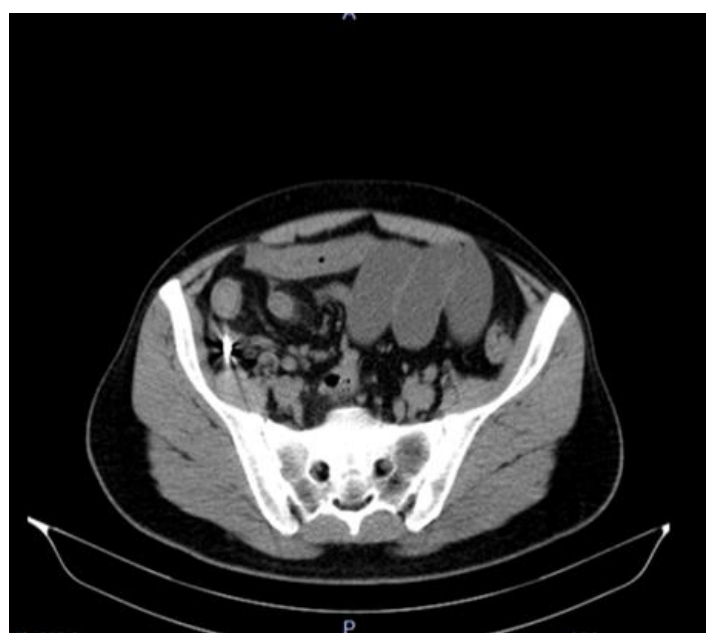

Figure 3. Intestinal obstruction and dilatation (Transverse Plane) 


\section{CONCluSion}

Inguinal herniation can cause small bowel obstruction. If the mass in the hernia blocks lumen, intestinal flow is obtructed. This is treated by manual intestinal reduction. Otherwise, patient needs to be operated. Both vitallity of intestinal segment in hernia sac and time between begining of the compliants and addmitance to the hospital are important. Strangulation is swelling and constriction of the blood supply of the herniated part, resulting in obstruction, gangrene and necrosis. Strangulation is twisting of intestines in hernia sac. Blood supply of intestine is sufficient [4]. In this case, strangulation wasn't thought due to CT findings and physical examination and we tried to reduce the hernia. After reduction, clinical singns of mechanical obstruction were spontaneously disappeared. This strenghtened our prediagnosis. Decline in leukocytosis and regression of clinical symptoms were indirect findings assured us that vitality of intestines were sustained. After 24 hours observation, elective operation was suggested to the patient. Simultaneous hernia repair procedure and orchiectomy were performed [4,5].

Other significant part of the case was metallic substances in CT scan. At first sight, the cause of these substanes couldn't be discovered. After further anamnesis, patient informed us about above conditions. At first anamnesis, patient didn't informed us about dropped tooth filling and consumption of hunted animal. We would like to emphasize the importance of anamnesis and sharing finding and results with patient.

\section{REFERENCES}

[1] www.tuik.gov.tr/veribilgi.do 11.2006/ Emet, Mücahit, et al. "Karın Ağrısı Olan Hastaya Yaklaşım."(2007)

[2] Zarvan, N. P., et al. "Abdominal hernias: CT findings." AJR. American journal of roentgenology 164.6 (1995): 1391-1395.

[3] Wechsler, R. J., et al. "Cross-sectional imaging of abdominal wall hernias." American Journal of Roentgenology 153.3 (1989): 517-521.

[4] Jenkins, John T., and Patrick J. O’Dwyer. "Inguinal hernias." BMj 336.7638 (2008): 269-272.

[5] Beitler, J. C., et al. "Complex inguinal hernia repairs." Hernia 13.1 (2009): 61-66. 